\title{
Challenges of gamification in software process improvement
}

\author{
Manal M. Alhammad ${ }^{1,2} \quad$ Ana M. Moreno ${ }^{1}$
}

\author{
${ }^{1}$ King Saud University, Riyadh, Saudi Arabia \\ ${ }^{2}$ Universidad Politécnica de Madrid, Madrid, \\ Spain \\ Correspondence \\ Manal M. Alhammad, King Saud University, \\ Riyadh, Suadi Arabia. \\ Email: manalhammad@ksu.edu.sa
}

\begin{abstract}
Software process improvement (SPI) initiatives have contributed for a long time to the progress of software development. However, the outcome of such initiatives has not always been as effective as expected. The literature of SPI points out that motivation of practitioners involved in SPI initiatives is a critical factor for its success. On the other hand, gamification has been perceived as motivational and has the potential to improve people's engagement. Thus, researchers have been recently investigating the impact of gamification in increasing the motivation and commitment of the organization members involved in SPI. Despite the novelty of the field, we see the integration of gamification and SPI as an opportunity of study and research. The aim of this paper is to investigate and report the state of the practice in this field, identifying key challenges faced by organizations wishing to incorporate gamification into their SPI initiatives. One of the main challenges identified is related to the complexity of gamifying SPI because it involves different organizational roles and aspects that may require designing different gamification strategies. Another relevant issue is the need of more empirical data to provide sound evidence about the real impact of gamification on improving SPI initiatives.
\end{abstract}

\section{1 | INTRODUCTION}

Organizations and companies around the globe invest tremendous efforts in improving their software development processes. ${ }^{1,2}$ The common assumption that the quality of software development process is directly linked to the quality of the developed software is what motivates research and practice in this area. ${ }^{3}$ The growing number of publications in the area of SPI, evidenced by a recent analysis of the literature, ${ }^{4}$ shows the significance of the field. Yet, even though most SPI methods and frameworks, such as Capability Maturity Model Integration (CMMI), have become well known among software engineering (SE) practitioners, studies show that many organizations, especially small-medium enterprises (SME), find the adoption of SPI frameworks to be challenging. ${ }^{5,6}$ In response, several endeavors are targeted to tailor standard SPI models or create custom SPI approaches for different purposes. For instance, lightweight techniques and integrated processes for process modeling and improvement have been developed in order to better address the demands of small and very small companies ${ }^{7,8}$ or to apply agile principles in the improvement process. ${ }^{9-11}$

In the field of SE, generally, practitioner lack of motivation was cited as one of the causes of software project failure. ${ }^{12}$ In the particular case of SPI, and since SPI is mainly a human endeavor, many studies have particularly investigated the people-management factors to understand why companies are failing to achieve the goals of improving their software process. ${ }^{13-16}$ Their key findings can be summarized as follows: "SPI has a higher chance of success in companies where practitioners experience high motivation for it". ${ }^{13}$ Additionally, Niazi insists upon the importance of being well informed about the SPI success factors in order to develop new or improved SPI strategies and investigates the impact of motivating 
the SPI initiative personnel as a success factor. ${ }^{17}$ Niazi claims that it is essential to know the impact of motivated practitioners on the success of SPI initiatives as it can help management prioritize and allocate resources properly. ${ }^{17}$

Since 2010, gamification has emerged as a powerful technique that can potentially increase motivation and engagement in people. ${ }^{18}$ The main principle of gamification is the use of game design and elements in nongame contexts. ${ }^{19}$ Although the first documented use of gamification was in 2008, gamification did not start to gain popularity in different sectors until early 2011 . It has been widely used in contexts such as marketing ${ }^{20}$ and education. ${ }^{21}$

In this context, employing gamification in SPI is seen as a research opportunity. As discussed later, some researchers have recently attempted to leverage the motivational factor of gamification to achieve higher success rates in SPI initiatives [P4][P7][P8]. Such efforts could be highly beneficial for increasing the favorable outcomes of SPI initiatives in organizations. As the literature points out, high motivation is one of the SPI success factors. ${ }^{13,17}$ Our aim is to investigate how gamification has been applied in the field of SPI. More precisely, our main objective is to understand if gamification can be effective in supporting SPI initiatives in terms of increasing the motivation and commitment of organization members. If so, it would be an interesting tool to promote motivation and thus improve the results of SPI experiences. To this end, we have performed a systematic mapping to get a snapshot of the field and to identify the current practices. While SPI has a long tradition, its integration with gamification does not. As mentioned above, gamification began to be used more widely in 2011. The results of the systematic mapping in terms of the number of primary papers are an indication of the novelty of this integration. As discussed, only eight primary papers were found. However, they have led us to develop a detailed overview of the field at this early stage. From this overview, we have identified a set of challenges that can open up interesting research and action opportunities. On the other hand, these results provide insights for professionals who are interested in rising to the challenge of gamifying their SPI initiatives at this very early stage.

The remaining of this paper is organized as follows. Section 2 reviews the related background in the field of SPI and gamification. Section 3 outlines the research methodology followed to perform the systematic mapping, including the definition of the research questions, the search and study selection processes for primary studies, as well as the data analysis. Section 4 reports the findings. Section 5 discusses the challenges identified. And finally, Section 6 concludes with summarizing key finding, challenges, and opportunities identified from this study.

\section{BACKGROUND AND RELATED WORK}

\subsection{Software process improvement}

SPI initiatives have been around for decades as a systematic approach to increase the efficiency of the software development process and to improve software quality. ${ }^{22}$ As SPI is a diverse field, various standards of SPI are available such as the Capability Maturity Model Integration (CMMI) or ISO/IEC 15504. A multitude of review studies summarizing the key trends and challenges in the field of SPI can be found in the literature. For example, Pino, García, and Piattini discuss SPI in the context of small companies ${ }^{23}$; Staples and Niazi explore the motivating factors for adopting CMMI, ${ }^{24}$ and Müller, Mathiassen, and Balshøj study SPI from the perspective of organizational change. ${ }^{25}$ More recently, Kuhrmann, Diebold, and Münch present a systematic mapping study on the state of the art in SPI. ${ }^{4}$ This study identifies a huge interest in studying SPI success factors, and particularly SPI in the context of very small enterprises adopting agile principles and practices. The study also underscored a lack of evaluation studies to investigate the current SPI models and frameworks, and due to the varying contexts, there is a shift towards proposing customized SPI models adapted to the different organizational environment. The hypothesis attributed to this trend of proposing customized SPI initiatives is that SPI always involves changes in the behavior of individuals and changes in the organization culture.

SPI projects normally require a considerable amount of effort and staff involvement as well as time and money. ${ }^{26}$ Therefore, SPI challenges and success factors have been extensively investigated in the literature. ${ }^{27-35}$ On one hand, studies that analyzed challenges in SPI suggest that in order to achieve the best results from SPI initiatives, four organizational elements must be part of the change which are, process, structure, management, and people. Focusing on the "people" element, O'Connor and Basri ${ }^{34}$ claim that people's attitude towards change, acquiring, and practicing new skills should be considered as the main dimension to improve in SPI. On the other hand, a key success factor in the SPI literature was found to be the commitment of the people involved in improving the software process. ${ }^{29}$ It is believed that SPI activities will sustain in the face of difficulties and barriers if an organization and its members from all levels are committed to SPI. ${ }^{35}$ In many SPI initiatives, the lack of commitment is appointed as the main cause of SPI failure or low process maturity. ${ }^{35}$ This may sound paradoxical, as the attempt to improve the software process should be triggered by an organization's aspiration and willingness to improve the efficiency of its process. The root cause of this lack of commitment issue remains ambiguous. Nonetheless, the SPI community acknowledges the need to deeply research the people aspect to better understand how to acquire their commitment.

Additionally, people management factors have been explored by Baddoo and Hall ${ }^{13}$ by focusing on motivators of SPI from the practitioners' view. Their findings suggest that even though each practitioner group has its own specific motivators, receiving rewards for SPI-related work was found to be a common motivator. Furthermore, they found that "top-down commitment" (ie, senior management support) and "visible success" (ie, evidence of the benefits of SPI) were the most cited motivators by software developers actively involved in SPI. Similarly, a more recent study 
by Passos et $\mathrm{al}^{16}$ found that despite practitioners' intrinsic motivation and enthusiasm about their work, they were demotivated by the lack of recognition they received for their efforts in improving software quality. Their findings also highlight the importance of management support for improving practitioner motivation. On a more general level, Yilmaz et al conducted a preliminary study on software practitioners to investigate social aspects of SPI, such as practitioner personality, claiming that the software process can be improved when personality types are taken into consideration. ${ }^{14}$ In sum, the SPI literature suggests that people management factors, particularly motivation, are crucial success factors in SPI.

\subsection{Gamification}

Gamification is defined as the application of game design and elements in nongame contexts as a motivational technique to encourage and facilitate new behaviors. ${ }^{19}$ It initially emerged in the business and marketing sectors implementing techniques such as loyalty cards and stamp books for increased customer loyalty and retention. Shortly after, gamification began to attract other domains such as education, ${ }^{21,36}$ health care, ${ }^{36}$ and innovation. ${ }^{37}$ The prevalence of gamification in various domains is thought to be based on the hypothesis that gamification is motivational and has the potential to increase user engagement. ${ }^{18}$ The benefits of gamification have been investigated in Hamari et al ${ }^{18}$ in which 24 empirical studies of gamification applied in different domains were analyzed. The main finding was that despite the need for more quantitative data, gamification appears to have a positive impact on motivating users towards adopting particular behaviors.

In the area of SE, some practices entail the enactment of different processes that are considered repetitive and time consuming. In this context, gamification is viewed as a promising technique that can potentially improve developers' interest and motivation in carrying out SE tasks. ${ }^{38}$ Pedreira et al conducted a systematic mapping on gamification in SE based on 29 studies. $^{39}$ The mapping revealed that software implementation is the area on which most studies focused, followed by software requirements, and few others in different areas such as project planning, and software testing. However, the highlight of this work was that gamification in SE is still at a very early stage and the evidence about the impact of gamification in this field remains inconclusive.

In this context, we see the study of gamification in the area of SPI an opportunity for research. We focus on analysing the field of gamification and SPI in order to understand the potential impact of gamification on process improvement, in terms of the potential benefits of gamification as a motivating factor as well as the major challenges and limitations of this integration.

It is important to note here that we specifically address the field of gamification, whereas game-based learning (GBL) and serious games are outside the scope of this paper. GBL deals with games designed for educational purposes. Serious games refer to the design of fully-fledged games for nonrecreational purposes. In contrast, gamification refers to merely employing game design principles and elements in nongame contexts.

\section{3 | METHODOLOGY}

In order to identify the main challenges of the integration of gamification and SPI, we performed a systematic mapping study guided by the recommendations of Petersen, Vakkalanka, and Kuzniarz. ${ }^{40}$ The literature review was performed during the last quarter of 2018 and covers publications in the field of gamification and SPI from January 2011 until December 2018.

\section{1 | Research questions}

Table 1 outlines the research questions established for this study. The aim is to explore how gamification has been exploited in SPI initiatives and to provide a current snapshot of the field to highlight the trends and gaps.

TABLE 1 Research questions

$\begin{array}{ll}\text { ID } & \text { Research question } \\ \text { RQ1 } & \text { What issues of SPI have been addressed by gamification? } \\ \text { RQ2 } & \text { How has gamification been implemented in SPI? } \\ & \text { RQ2.1. What gamification processes or approaches, if any, have been followed to incorporate gamification in SPI? } \\ & \text { RQ2.2. What gamification elements/components have been used? } \\ \text { RQ3 } & \text { What is the evidential impact of gamification on SPI? What aspects of SPI have been affected or improved? }\end{array}$


RQ1 aims to understand the purposes or goals behind gamifying SPI. Specifically, it aims to understand the issues of SPI which motivated SE practitioners to use gamification. RQ2 deals with how gamification was put into practice in SPI. This research question is divided to three subquestions: RQ2.1 focuses on exploring the different methodologies and gamification processes followed to gamify SP; RQ2.2 aims to outline the game elements incorporated into the gamified SPI process; and RQ2.3 attempts to identify the tools developed or used to apply gamification to SPI. Finally, RQ3 explores the evidence about the impact of gamification on SPI and whether gamification is effective in addressing its goals in SPI. This can be helpful to understand whether gamification has positive, negative, or no effect on the overall SPI process.

\subsection{Search process and study selection}

The search process employed in this study was designed to achieve the maximum level of coverage. The search string was designed as comprehensive as possible, while limiting the keywords to the scope of the study. The search string was constructed using two major terms: "gamification" and "software process improvement". The result was: "("software process improvement" OR SPI) AND (gamification OR gamify OR gamified OR gamifying OR gameful)". We used the general term "software process improvement" instead of specific SPI frameworks and methods (eg, CMMI) to achieve a broader coverage. IEEE Xplore, ACM DL, SCOPUS, Web of Science (core collection), and ScienceDirect were selected as our primary sources based on the guidelines of. ${ }^{40}$ The search string was applied on meta data. We also applied backward snowballing by tracking the references of potentially relevant papers. However, due to the small size of literature, no further publications were found. Table 2 provides a summary of the search results per database.

The selection of primary studies was done through two screening stages: reading of titles and abstracts to identify potentially relevant studies, and reading the full text to make a decision of inclusion or exclusion. We essentially included studies that deal with gamifying SPI and excluded the following:

1. Studies dealing with games, serious games, or GBL or do not explicitly use gamification.

2. Studies whose full text is not accessible.

3. Studies not written in English.

4. Summaries or reviews of conferences.

5. Studies that are shorter versions of other studies.

TABLE 2 Summary of search results

\begin{tabular}{ll} 
Database & Search Results \\
\hline IEEE Xplore & 6 \\
\hline ACM Digital Library & 0 \\
\hline SCOPUS & 25 \\
\hline Science Direct (in computer science) & 1 \\
Web of Science (core collection) & 14 \\
\hline Total & 46 \\
\hline
\end{tabular}

TABLE 3 Rationale for excluded studies

Reason for Exclusion
Duplicates
Not in the software process improvement (SPI) context
No explicit use of gamification, or using game-based learning or serious games
Full text not accessible
Not written in English
Summary or reviews of conferences
Studies that are a shorter version of other studies
Total excluded
Total included


As a result, illustrated in Table 3, eight papers were included as primary studies. Five of these papers share the same first author but are related to different studies and were published over 5 years. The full list of primary studies can be found in the Appendix.

\section{3 | Data extraction and analysis}

A data extraction form, depicted in Table 4, was designed to capture the necessary data for answering the research questions. The key fields are:

- Research type: the classifications for this field were adopted from Wieringa et al ${ }^{41}$ in order to record the type of research reported in the primary studies. The classification categories are evaluation research, validation research, solution proposal, experience paper, philosophical paper, or opinion paper.

- The objective of applying gamification in SPI to record the challenges in SPI that lead the authors to gamify SPI.

- Gamification approach, recording series of steps or actions, if any, followed to gamify SPI.

- Gamification elements, recording gamification dynamics, mechanics, and components incorporated in gamified SPI processes. For this field, we used Werbach and Hunter's definition in Werbach and Hunter ${ }^{42}$ as our main reference.

- Type of implementation, recording the supporting tools or platforms, if any, that were developed or used as part of the gamified experience.

- Gamification impact, to record any empirical evidence about the impact of applying gamification in SPI.

The data extraction process was mainly performed by the first author and reviewed by the second author through checking the validity of the extracted data. We followed the thematic analysis approach by Cruzes and Dyba. ${ }^{43}$ In this stage, text segments corresponding to the research questions were captured and recorded in the data extraction form for the analysis process afterward. However, for some research questions, the process was not straightforward. For example, we sometimes had to code different text segments across different papers to capture data related to one RQ or one field in the data extraction form then trying to synthesize the pieces of text to form an answer or categorization criteria for the RQ. For instance, we had to synthesize and summarize the data collected for RQ1 about the challenges of SPI that lead the authors to use gamification in order to generate common categories that includes all challenges. The next step involved analyzing the captured data by looking for themes and patterns across papers. However, due to the lack of literature found, some papers failed to provide clear information about certain fields, thus, making the analysis process seem difficult. Nevertheless, this has led to some interesting and insightful findings as discussed in the next sections.

\section{\begin{tabular}{l|l}
4 & RESULTS
\end{tabular}}

\section{\begin{tabular}{l|l}
4.1 & General results
\end{tabular}}

The first primary study that introduced gamification in SPI dates back to 2012 proposing to use gamification as a solution in the context of SPICE [P1]. In 2014, Herranz et al [P3] presented a conceptual framework for gamifying SPI and conducted a theoretical validation to assess the

TABLE 4 Data extraction form

\begin{tabular}{|c|c|c|}
\hline Data Item & Value & RQ \\
\hline Study ID & First author's last name + year of publication & \\
\hline Article Title & Name of the article & \\
\hline Author Name & Set of names of the authors & \\
\hline Year of Publication & Calendar year & \\
\hline Venue & Name of publication venue & \\
\hline Type of Research & Validation research, evaluation research, solution proposal, experience paper, philosophical, opinion paper. & \\
\hline OBJECTIVE & What are the challenges of SPI that have been addressed by gamification? & RQ1 \\
\hline APPROACH & What approach has been followed to gamify SPI? & RQ2.1 \\
\hline ELEMENTS & What gamification elements have been used? & RQ2.2 \\
\hline TOOLS/TECHNOLOGIES & What tools or supporting technology has been employed to assist in gamifying SPI? & RQ2.3 \\
\hline IMPACT & What is the impact of gamifying SPI? on which aspects? What was improved or affected? and to what extent? & $\mathrm{RQ3}$ \\
\hline NOTES & Any special notes the authors wanted to record for each study & \\
\hline
\end{tabular}


applicability of the proposed framework. The year after, the technical implementation of the aforementioned framework was presented as a platform called "Gamiware," and a pilot study was carried out [P6]. Although the number of publications in this field is small, the year 2016 shows a peak with three validation studies conducted to investigate the impact of gamification on SPI. Yilmaz and O'Connor [P8] performed a validation study by surveying software practitioners in an SME as part of an organizational transformation process that involved adopting a gamified Scrumban process to improve the development process. Also, Ruiz, Trinidad, and Calderón [P7] conducted a pilot study to assess the validity of their own gamification proposal using a post-experience questionnaire. Moreover, Herranz et al [P4] employed a qualitative research through interviews that involved experts from gamification and SPI to further assess the validity and relevance of their framework presented in [P3] and [P6]. In 2017, [P5] deployed the gamification framework (Gamiware) in a small company to confirm the validity of the framework. Finally, in 2018, an experience paper was published by Herranz and Colomo-Palacios [P2] to reflect back on their gamification experience with Gamiware, mainly discussing the success factors, or enablers, of gamification initiatives in SPI.

\subsection{RQ1-what issues of SPI have been addressed by gamification?}

RQ1 aims to outline the objectives behind employing gamification in SPI initiatives. According to the data collected from primary studies, the issues of SPI that literature attempts to address using gamification can be grouped into the four following categories:

- Engagement and motivation: The lack of motivation towards SPI initiatives was discussed in all primary studies as the main challenging issue. For instance, [P1] believes that motivation of top managers is the key element for the success of SPI initiatives, particularly in organizations where different levels of management exist. They also emphasize on the need to foster a new kind of transformation that conform with generation $Y$ (ie, millennials) that expects clear goals, social visibility, and rewards as a mean to increase their motivation in the workplace. A similar perspective was presented by Herranz et al in [P3] where the authors analyze the success factors for SPI and include the motivation of top managers as a vital factor to attain their commitment towards implementing SPI initiatives. They argue that the lack of top managers motivation, and thus commitment, can be a major demotivator for software developers which can significantly affect the success of SPI. They also highlight the importance of orienting (aligning) the desired outcome of SPI initiatives together with business objectives in motivating top managers to make the benefits more visible and clearer. With less focus on top managers, Ruiz, Trinidad, and Calderon [P7] and [P8] also stress on the lack of motivation among software practitioners in the design and implementation of SPI initiatives that may often lead to a lower level of commitment, which is another common issue in SPI.

- Commitment: Without constant and firm commitment towards SPI, there can be some costly waste of effort and higher chances of failure. The issue of commitment is mostly highlighted in [P3] and [P7]. Both studies highlight the importance of long-term commitment to leverage SPI benefits to the maximum level. [P3] have underlined the commitment of top managers as the most highlighted success factor of SPI according to the literature of SPI, and that management support is a valuable element in avoiding critical success barriers such as organizational hierarchies and politics. On the other hand, [P7] appointed lack of commitment as the major cause leading to the failure of SPI initiatives which may sound paradoxical since an organization that adopt SPI activities in its processes is primarily attempting to improve these processes, yet lack of commitment emerges as a common issue.

- Communication: Visibility and clarity of goals within an organization is considered a challenge in SPI initiatives. More specifically, feedback of achievements and progress indicators regarding process improvement is highlighted by [P1] as two areas of improvement in SPI that can benefit from using gamification. [P8] suggest that gamification can address communications issues in SPI by improving social interactions among software practitioners using interactive game elements as well as increasing the perception of progress during the improvement process. Similarly, the importance of having clear, relevant, and visible goals exchanging between management and developers has been underscored in [P3].

- Change management: The only primary study that addresses this issue is [P3]. The authors claim that SPI success requires the involvement and coordination of different divisions across an organization. Staff involvement, allocation of resources, organization culture, and politics are among a list of factors related to organizational transformation that can play a role in the success of SPI. Misalignment between the different parts of an organization can cause failure to effectively manage the SPI program.

\section{3 | RQ2-how has gamification been implemented in SPI?}

The aim of RQ2 is to understand how gamification was put into practice in the context of SPI. For that reason, we address three aspects: the approaches or processes followed by primary studies to gamify SPI (RQ2.1), the game dynamics, mechanics, and components integrated into the gamified SPI process (RQ2.1), and the details of any tools used or technical implementation (RQ2.3). Table 5 summarizes the data gathered for 
TABLE 5 Summary of the information gathered about gamification implementation in the context of SPI

\begin{tabular}{|c|c|c|c|c|}
\hline ID & SPI Issues & Approach & Gamification Elements & Supporting Tools \\
\hline$[\mathrm{P} 1]$ & $\begin{array}{l}\text { Motivation and } \\
\text { communication. }\end{array}$ & $\begin{array}{l}\text { No gamification approach was } \\
\text { followed. }\end{array}$ & $\begin{array}{l}\text { Dynamics: Progression. } \\
\text { Mechanics: Feedback, Rewards, } \\
\text { Challenges. } \\
\text { Components: Points, Badges. }\end{array}$ & $\begin{array}{l}\text { Not applicable since this study } \\
\text { is categorized as opinion } \\
\text { paper. }\end{array}$ \\
\hline [P3] & $\begin{array}{l}\text { Motivation (with a focus on } \\
\text { top managers), } \\
\text { commitment, } \\
\text { communication, and } \\
\text { change management. }\end{array}$ & $\begin{array}{l}\text { The authors refer to Werbach } \\
\text { and Hunter }{ }^{42} \text { as their main } \\
\text { guide in designing their } \\
\text { gamification framework. }\end{array}$ & No details were provided. & No details were provided. \\
\hline$[\mathrm{P} 6]$ & $\begin{array}{l}\text { Motivation (with a focus on } \\
\text { top managers), } \\
\text { commitment, } \\
\text { communication, and } \\
\text { change management. }\end{array}$ & $\begin{array}{l}\text { The authors refer to Werbach } \\
\text { and Hunter }{ }^{42} \text { as their main } \\
\text { guide in designing their } \\
\text { gamification framework. }\end{array}$ & $\begin{array}{l}\text { Dynamics: progression, relationships. } \\
\text { Mechanics: Competition, cooperation, } \\
\text { challenges, rewards, feedback. } \\
\text { Components: points, badges, levels, } \\
\text { leaderboards, achievements. }\end{array}$ & $\begin{array}{l}\text { A gamification platform called } \\
\text { "Gamiware" was } \\
\text { implemented to represent } \\
\text { their gamification } \\
\text { framework. }\end{array}$ \\
\hline [P8] & $\begin{array}{l}\text { Motivation and } \\
\text { communication. }\end{array}$ & $\begin{array}{l}\text { The authors adopted the "6D" } \\
\text { gamification framework } \\
\text { designed by Werbach and } \\
\text { Hunter. }{ }^{42}\end{array}$ & $\begin{array}{l}\text { Dynamics: progression; } \\
\text { mechanics: rewards, feedback; } \\
\text { components: points, badges, quests, } \\
\text { avatars, achievements }\end{array}$ & No details provided. \\
\hline
\end{tabular}

RQ2. The primary papers [P2], [P4], [P5] were not mentioned in Table 5 because they are validation and evaluation studies of the same framework proposed in [P3] and [P6] and do not provide additional details related to RQ2.

In general, although most primary studies follow the "6D" gamification framework of Werbach and Hunter, ${ }^{42}$ the details of how each primary study carried out the gamification of SPI show that there is no rule of thumb when it comes to applying gamification in SPI. Like the "6D" process, a gamification process can be generally flexible enough to be customized to different organization with different goals and processes, and in different contexts.

In the case of [P3], the authors cite the "6D" gamification framework as their main source to guide the design of their own gamification framework. However, they tailored the $6 \mathrm{D}$ process to fit their objectives by dividing the process into two stages. In the first stage, a high-level gamification proposal is designed to plot a general roadmap for implementing gamification in an organization with consideration to different groups of software professionals. The outcome of this stage served as guidelines to the lower level details of the framework. In the second stage, the high-level proposal is customized to fit each group of software professionals in their organization. This personalization process involved defining specific techniques for each group of professionals such as senior management, project managers, and developers. They claim that this approach should achieve higher motivation levels according to Baddoo and Hall, ${ }^{13}$ who performed an analysis on the role of human factors in the success of SPI initiatives. However, [P3] in this stage focused on common motivators that can be boosted by gamification among the groups of software professionals in their organization.

Furthermore, the gamification strategy designed in [P7] was also based on following the "6D" guidelines. The authors of [P7] started by performing an initial evaluation of their case and defining their aim of using gamification in SPI. Then, they defined their target audience (ie, software engineers) and what drives their motivation. Notably in this step, they suggest that change and challenge fuels software practitioners' motivation. After that, they define the activities cycle that will be gamified within SPI processes. For example, challenging software engineers with SPI problems of higher complexity disseminated throughout alternative cycles. Finally, they decided to add a fun factor to the gamification process through instant feedback and collaborative culture in the form of a mobile application.

Similarly, [P8] reported that they also followed the " $6 \mathrm{D}$ " gamification framework to guide the gamification experience of a Scrumban process. Again, [P8] did not detail the steps and actions that were taken to adapt the "6D" framework to their case. Finally, extracting details regarding the gamification approach in [P1] was not applicable since the study is classified as "opinion paper," where the author did not report an attempt to design a solution to gamify SPI. However, a background analysis on gamification and SPI provided suggestions and insights on how gamification can be leveraged in the field of SPI, and why certain game elements can be effective in motivating people in the workplace towards process improvement. 
RQ2.2 outlines the game dynamics, mechanics, and components used in primary studies. We followed Werbach and Hunter's definition of dynamics, mechanics, and components. ${ }^{42}$ Game elements that are not explicitly mentioned in the primary studies were not considered, thus, avoiding inferring any information and only recording the ones mentioned by the authors. The fourth column of Table 5 sums up the gamification elements implemented in gamifying SPI. Badges were found to be the most frequently used game component, integrated in all primary studies' gamification proposals or experiences. The rationale behind using badges, or specific elements over others, was not clearly discussed in primary papers. However, [P1] reported that collectible badges are trending in the literature of gamification due to their persistence nature. In other words, unlike momentary rewards (eg, points) that are given upon completion of a certain task, badges can sometimes be viewed as permanent rewards that can represent one's achievements or expertise over the entire time (eg, a whole level of a certain processes or a certain skill). The use of badges in primary studies was not clearly explained in terms of what kind of activities or tasks within SPI can benefit the most from awarding badges. [P1] suggested that awarding badges upon obtaining process capability or organizational maturity levels in the context of SPICE can be effective in achieving SPI goals.

In terms of game mechanics, feedback was the most cited technique in primary studies. The importance of frequent feedback is to provide software practitioners with an updated status of their progress and how well they are doing in a particular task [P1]. To a deeper extent, [P6] employed game components, such as progress bars and leaderboards, as a mean to provide feedback to developers about their progress in a certain task, as well as for senior management to have full knowledge about the overall progress of a project. Moreover, they emphasized on the importance of providing real-time feedback to orient teams and individuals involved in the SPI initiative to adopting best practices. As for [P7] and [P8], both studies reported the utilization of a feedback element in the gamification of SPI, but they lack details about in what form feedback was provided or how it was implemented.

RQ2.3 attempts to identify the tools and platforms used, or developed, in the process of gamifying SPI. The analysis phase showed that only two studies provided some information about the technical implementation of gamification in SPI. [P6] developed a gamification platform called "Gamiware"-aimed to increase motivation in software projects-that was implemented as an open source Software as a Service (SaaS) to represent their gamification framework. The tool was designed to be customizable and flexible. For example, each organization can code their own business, software, or SPI objectives, identify groups of software practitioners involved in the gamified SPI, and define the contribution of each task to the business or software objectives. On the other hand, [P7] developed two tools to implement their gamification strategy: (a) "OpenBadgesUCA" to send the user's badges to their account in Mozilla's OpenBadges platform (openbadges.org) and (b) "GamAnalyze" to analyze the data collected by OpenBadgesUCA to assess the effectiveness of the gamification experience.

\subsection{RQ3-what is the evidential impact of gamification on SPI?}

RQ3 focuses on exploring the evidence reported in primary studies about the impact of gamification on SPI. Overall, due to the small number of publications in the literature, the analysis does not provide clear and conclusive results. However, the results of the different evaluation and validation studies conducted in the primary papers are worth mentioning. Such information can be useful for software practitioners who plan to gamify their SPI to discover the various benefits and limitations of gamification in SPI and to highlight the lessons learned from previous experiences. A summary of the reported impact of gamification on SPI is depicted in Table 6.

TABLE 6 Summary of the reported impact of gamification on SPI

\begin{tabular}{|c|c|c|}
\hline ID & Reported Impact of Gamification on SPI & Research Type \\
\hline$[\mathrm{P} 1]$ & No impact reported. & Opinion paper \\
\hline$[\mathrm{P} 3]$ & $\begin{array}{l}\text { A low degree of consensus was observed among gamification and SPI experts regarding the critical success } \\
\text { factors (CSFs) which the authors identified for gamifying SPI. }\end{array}$ & Theoretical validation \\
\hline$[\mathrm{P} 6]$ & $\begin{array}{l}\text { Participants reported that their motivation level is increased with regard to performing SPI related tasks. } \\
\text { Most participants were satisfied with their performance and felt competent in performing SPI tasks. } \\
\text { The participants also reported lack of fun using the tool "Gamiware" and felt that they are under pressure. }\end{array}$ & Validation \\
\hline [P4] & $\begin{array}{l}\text { No impact reported. The gamification framework was validated by gamification and SPI experts and concluded } \\
\text { that it "sustains its relevance and applicability in SPI initiatives." }\end{array}$ & Validation \\
\hline$[\mathrm{P} 5]$ & Significant improvement in the execution of software tests. & Evaluation \\
\hline$[\mathrm{P} 2]$ & No impact reported. & Experience \\
\hline$[\mathrm{P} 7]$ & $\begin{array}{l}\text { Inconclusive report on impact. Accomplishment and empowerment were the best rated indicators, while } \\
\text { social influence and curiosity were the lowest. }\end{array}$ & Validation \\
\hline$[\mathrm{P} 8]$ & $\begin{array}{l}\text { Increased knowledge and level of understanding about the software development process. Improved } \\
\text { performance among software developers. }\end{array}$ & Evaluation \\
\hline
\end{tabular}


To begin, six primary studies performed some kind of assessment to their gamification experience, whether it was validation (in a controlled or laboratory setting) or evaluation (in a real setting). Subsequently, for the purpose of reporting a comprehensive review about the impact of gamification on SPI, the results of each study will be discussed individually below:

- [P3]: Herranz and his colleagues started evaluating their gamification framework by performing a "theoretical validation." The authors conducted the theoretical validation by creating a set of "critical success factors" (CSF) that they determined from an in-depth review of the proposed solution/framework. The ground of following this method in identifying CSFs, while they are usually obtained from analyzing existing literature, is attributed to the absence of studies in the field of gamification and SPI. The validation was performed through a focus group of experts in gamification where the participants rate the validity of each CSF. In general, a low degree of consensus was observed regarding the critical success factors of the framework. The authors attributed this to the novelty of the field, and the sample of participants in terms of size and quality. Nevertheless, the authors suggested using these CSFs as guidelines for the implementation of the framework and emphasized on the necessity for an empirical validation. In sum, no evidential impact of gamification on SPI has been reported in this phase of the study as the framework was not built and tested in a real environment nor a laboratory/controlled setting.

- [P6]: A pilot study was conducted to validate a platform developed to gamify SPI, ie, "Gamiware." The aim of this validation study was to measure the impact of gamification on software practitioners' motivation, as well as testing the applicability of the tool in SPI initiatives. A total of 24 SE students participated in this study. The platform received both positive and negative responses. In terms of motivation, participants reported that their motivation level is increased with regards to performing SPI-related tasks. Similarly, most participants were satisfied with their performance and felt competent in performing SPI tasks. On the contrary, the participants also reported lack of fun using the tool "Gamiware" and felt that they are under pressure. This is a key factor to consider that may, or may not, have affected the reliability of the overall results of this validation study.

- [P4]: The authors here conducted a validation study through semistructured and structured interviews with gamification experts and SPI experts, respectively. The validation was done in two phases, the first phase was aimed to validate their framework from a gamification perspective with the help of gamification experts. In this phase, the authors performed two rounds of interviews. Round one was aimed at understanding how gamification is applied in organizations, which involved general questions about gamification, not specific to the framework.

Round two was aimed at validating the framework itself, getting the experts feedback about it. The second phase involved validating the framework from the perspective of SPI experts to assess the practicality deployed in SPI initiatives. The validation was done through webbased interviews. To our understanding, this validation was done after modifying the framework based on the feedback gathered from the gamification experts. The results of the two phases of validation, however, were not discussed thoroughly in the paper. A summary of the feedback they received as well as an analysis of the responses was missing. Quoted responses from gamification experts were provided but without presenting a discussion or analysis and how it relates to the validity of the framework. In other words, it was difficult for the reader to understand if the framework was valid or not from the experts' perspective, given only some parts of the interview responses, without raw data nor an analysis from the authors' perspective.

Nevertheless, in the conclusion, the authors provided a brief summary of the results claiming that the framework "sustain its relevance and applicability in SPI initiatives." They finally highlight the limitation of this type of validation dealing with qualitative data and the need to empirically "evaluate" the framework in a real environment.

- [P5]: In this publication, Herranz et al present a case study where they evaluate the framework in a small-medium company. The company's aim of using gamification is to improve the overall execution of software testing, that is generally affected by a lack of time and resources. Accordingly, the evaluation process was conducted to investigate whether the motivation of software developers towards software testing can be improved through carrying out SPI activities using the gamification framework. Although the authors initially intended to use "Gamiware" to support the evaluation process, they found that the tool would put in additional complexity to the process. Accordingly, they decided to carry out the evaluation process by essentially applying the framework itself into the existing software the company used, which is Gitlab. Even though they note that Gitlab has a public API, which provides the opportunity to develop some kind of plug-in, they opted not to implement any tool to "reduce resistance to change as much as possible." Therefore, they designed a gamified workflow where developers were awarded points manually by the manager based on reviewing executed tests by each developer. The results of the evaluation process showed a significant improvement in the execution of software tests. The motivation was measured by a questionnaire of a 5-point Likert scale given to the participants at the beginning and at the end of the evaluation process to measure the variation level of motivation before and after gamification. No further details were provided regarding the results of the questionnaire either in terms of qualitative data, such as the responses of the questionnaire, or quantitative data such as the number of executed tests before and after the experiment.

- [P7]: A pilot study was conducted which involved surveying 10 volunteers of SE students and professors. A postexperience questionnaire revealed a diversity in participants responses towards the motivational facet of gamification. To measure motivation, the authors cite Chou's proposal $^{44}$ that defines seven indicators of a motivated individual: accomplishment, empowerment, ownership, curiosity, control, creativity, and social influence. They found that accomplishment and empowerment were the best rated indicators, while social influence and curiosity were the lowest. They attribute the low rating of curiosity to the fact that their tool did not implement any uncertain or random event, which in 
principle what triggers curiosity. As for social influence, although the tool supports sharing achievement via twitter, not all participants can be profiled as competitors. Finally, the indicators of ownership and control were rated relatively positive as the gamification strategy they designed allowed participants to create their own SPI solution. In sum, the impact of gamification on SPI is inconclusive in this case, and empirical studies on a wider sample in a real environment are necessary.

- [P8]: Yilmaz and O'Connor performed an evaluation study using three surveys over two stages of software process transformation in a smallmedium company. The company was aiming to improve its software development process by gradually transitioning from scrum to scrumban, and then from scrumban to gamified scrumban. An initial survey was conducted to measure with a focus group to investigate the issues in the current software development process. Following that, the company changed their software process from scrum to scrumban, and then 6 months later they surveyed software engineers again to get their feedback and measure their overall performance and satisfaction. Based on the data gathered from the previous survey, a gamified scrumban process was introduced and adopted to address the concerns and suggestions made by the software developers. Six months after integrating gamification to the development process, the same survey was conducted once again to measure the impact of gamification on improving the developers' motivation and social interaction. The results showed an increased knowledge and level of understanding about the software development process as well as an improvement of performance among software developers.

\section{5 | CHALLENGES OF GAMIFICATION IN SPI}

Although the results of this systematic mapping study cannot be conclusive, they provide a comprehensive overview of the field in this early stage and have highlighted interesting challenges as groundwork for further research. Additionally, these challenges represent critical points to be considered by practitioners who decide to rise to the effort of improving their SPI initiatives at this early stage. Below, we discuss the identified challenges:

- Defining the target audience of gamifying SPI: The literature of SPI clearly distinguish the different roles in SE teams in general, and in SPI initiatives in particular, in order to effectively manage the different SPI initiatives. ${ }^{22,45}$ It is clear that the involvement of all the different levels of an organization in SPI initiatives is critical for its success. When gamifying SPI, it is important to clearly inspect and identify which roles will be involved in the gamification experience. In this context, all primary papers mention the importance of motivating senior management in the gamification process. However, with the exception of [P6], none of the papers performs a detailed analysis of the different roles involved in the gamification process. And even though the role of senior management and its high relevance was specially recognized in [P6], this role was not explicitly addressed when applying their gamification framework. The "Gamiware" tool they developed included different accounts to reflect the different roles, but only the developer and the project manager role have integrated gamification techniques.

- Defining the target behavior or expected outcome from gamifying SPI: Apart from motivation, primary papers aimed to improve communication among management and developers using gamification, asserting again on the importance of having all different levels of an organization involved in SPI. Yet in this case, the benefits of gamification in improving communication were more apparent. Gamification can help in providing a transparent SPI environment where management and software engineers can keep track of progress and goals achieved through simply implementing indicators like progress bars. To effectively gamify SPI, it is critically important to establish a well-defined goal and common understanding among the practitioners involved in SPI. ${ }^{42}$ The more precise the goals are, the more likely for the gamification process to succeed.

- Designing different gamification strategies for the different organizational roles: Due to the different nature of SPI, gamifying the software process in an organization may require more effort than gamifying other processes in the SE field that generally involves participants of similar background or characteristics. For instance, when gamifying SE education, the people involved in the gamification experience are, generally, students of the same level of background and are driven by similar motives. ${ }^{45}$ Regardless of whether all the different levels of an organization participate in the SPI initiative, the difference between the various roles involved in the gamification experience should be analyzed and taken into consideration. ${ }^{42}$ This may require designing different gamification strategies and techniques tailored to each group of practitioners because not all players (ie, people involved in the SPI initiative) in this case react the same way nor have similar motives.

- Choosing the appropriate approach to implement gamification: Most primary studies followed the "6D" general gamification framework. ${ }^{42}$ However, they lack details about the concrete actions and steps taken to apply this framework into the context of SPI. Generally, we noticed that there is a missing alignment between each step of the gamification process and the targeted SPI aspect or activity. For example, we were unable to identify the grounds for selecting particular game mechanics or components, given the different aspects of SPI to be gamified. This challenge is not limited to the SPI and gamification field. In the agile and gamification field, Alhammad and Moreno also found that irrespective of whether or not a gamification approach was used, most studies do not discuss the grounds for selecting the gamification elements, neither do they address which of the gamification elements were targeted to achieve which particular goal within agile software development. ${ }^{46}$ This scenario is related to what is known as "pointification" in the gamification literature. Pointification is described as the sole use of points to 
reward participants and badges to create rankings, overlooking other gamification design principles that are helpful for achieving the main goal of the gamified experience. ${ }^{48}$ Providing more empirical data, in the form of examples or case studies, would help to illustrate what type of SPI activities or practices were subject to gamification, or what particular game elements were selected to incentivize a particular behavior.

- Lack of empirical data: It is important to conduct more empirical studies in the field to gather more data and generate more reliable results and conclusions for the SPI community. Even with the information provided in primary studies about the effectiveness of gamification in SPI, the data are hard to generalize given that the majority of experiments were not conducted in a real organizational setting. The type of metrics used in primary studies to measure the effectiveness of gamification is another issue. Although all primary studies report some positive impact of gamification, the reported impact is based on interviews and surveys, which are prone to subjectivity. No measurable improvement in the software process was reported. This made it difficult to measure the significance of the impact of gamification beyond claims made by the primary paper authors. Even though qualitative data are useful to provide key information about the gamified experience, they should be combined with quantitative metrics to provide a clear definition of what is considered "improvement" in the software process.

- Managing the additional effort introduced by gamifying SPI: Gamifying a process should not impose a significant change that entails learning about gamification or completely transforming the way people work. In the case of [P5], they opt for not using "Gamiware" (the tool they developed to gamify SPI) in the evaluation study to reduce the "resistance to change." They assumed that the tool would impose additional complexity to the evaluation process as the participants would first have to learn about the tool and how it works and then switch from "Gitlab," the tool they are familiar with and been using for years, to "Gamiware." We take the view that introducing gamification in a gradual fashion can reduce the resistance to change and cognitive workload necessary to gamify a process. Buisman and Van Eekelen ${ }^{47}$ describe this idea as a "low-intensity gamification" approach where gamification is introduced gradually starting with no new activities and merely integrating gamification elements into existing processes. On the contrary, "high-intensity gamification" entails creating new processes and activities as part of the new gamified experience. Besides, the introduction of gamification as a separate entity, when gamification is concerned with "integration in" or "combination with" the existing process, would solely increase the effort necessary to improve the software process and thus distract people with new rules and new elements blurring the essence of SPI as a whole.

\section{6 | CONCLUSION}

This mapping study has highlighted that gamification in SPI is in its early stages, and there is ample opportunity for detailed study and exploration. Given the nature of software development and its human-intensive features, the literature of gamification in SE has shown that gamification can be potentially effective in motivating software developers and improving their overall performance. ${ }^{39}$ In the case of SPI and gamification, our analysis shows that there are several important challenges and limitations that can open up interesting research and action opportunities. Those challenges also point to critical issues that need to be carefully considered by practitioners who aim to gamify SPI initiatives.

The resulting challenges mainly focus on the complexity of gamifying SPI. Sources of complexity are the different organizational roles and gamification strategies, the lack of a specific SPI gamification framework, and the need for more empirical data to establish the specific impact of gamification on the different roles involved in SPI. As we have discussed, some of the challenges identified are not restricted merely to the SPI domain, but have also been found to apply to gamification in $\mathrm{SE},{ }^{39}$ gamification in SE education, ${ }^{45}$ and gamification in agile. ${ }^{46}$

We should emphasize the need for more empirical research to obtain reliable data about the benefits and drawbacks of gamifying SPI. Further research should be conducted to explore the impact of gamification on improving the software process in terms of measurable benefits, such as improvement in production analytics or cycle time in agile teams, that can be visible to senior management. Similarly, information about the maturity level of the organizations that gamified their SPI initiatives can provide an insight into how significant the maturity level is with regard to the gamification experience. The effectiveness of gamification in improving the commitment of particular roles in an organization, such as senior management, should also be studied more empirically.

Note that the introduction of gamification should not make major changes to the SPI initiative if the gamification experience is to be successful. Change resistance is common in any environment, particularly when people are familiar and comfortable with the way they work and the tools they use. In situations where change is necessary, gamification can be of benefit when introduced gradually, starting with no new activities or tools, but merely deploying gamification elements such as points, badges, and leaderboards (PBLs). ${ }^{42}$ When used right, PBLs can serve as a starting point to design a gamified process. However, to get the maximum benefit of gamification, a gamified system should move beyond PBLs.

Additionally, gamification techniques, such as levels and badges, should be used to provide participants with a sense of progression in order to stave off boredom and keep the practitioners engaged in the SPI initiative. ${ }^{42}$ Levels in the case of SPI can provide the practitioners with a perspective about the development and advancement of the SPI initiative. Badges, on the other hand, are an effective way of progress recognition by visually representing a practitioner's skills and achievements.

Furthermore, it is important to identify and analyze which roles will be involved in the SPI gamification experience. ${ }^{38}$ Not all people are motivated in the same way. Accordingly, once the roles have been specified, the factors that motivate and demotivate each group of practitioners should be analyzed. Therefore, the design of different gamification techniques involving different gamification elements may be required. 
Even though gamification is effective at motivating practitioners, it can produce results that are not helpful or are irrelevant to the goals or expected outcomes of the SPI initiative. Therefore, it is equally important to clearly establish the objectives of using gamification and align gamification with the goals of the SPI initiative. This would not only keep the SPI team focused on working towards achieving the goal of SPI, but it can also help in raising commitment and engagement from senior management.

In sum, even though gamification is a rather simple idea, its application in a complex area like SPI requires in-depth analysis and preparation. In this paper, we have highlighted the main challenges of this process and have discussed some recommendations for dealing with these questions.

\section{REFERENCES}

1. Niazi M, Babar MA, Verner JM. Software process improvement barriers: a cross-cultural comparison. Inf Softw Technol. 2010;52(11):1204-1216.

2. Samalikova J, Kusters RJ, Trienekens JJM, Weijters A. Process mining support for Capability Maturity Model Integration-based software process assessment, in principle and in practice. J Softw Evol Process. 2014;26(7):714-728.

3. Iqbal J, Ahmad RB, Nasir MHNM, Niazi M, Shamshirband S, Noor MA. Software SMEs' unofficial readiness for CMMI ${ }^{\circledR}$-based software process improvement. Softw Qual J. 2016;24(4):997-1023.

4. Kuhrmann M, Diebold P, Münch J. Software process improvement: a systematic mapping study on the state of the art. PeerJ Comput Sci. 2016; 2(2012):62.

5. da Silva GC, de Figueiredo Carneiro G. Software process improvement in small and medium enterprises: a systematic literature review. In: Information Technology: New Generations. Springer; 2016:603-613.

6. Sánchez-Gordón M-L, Colomo-Palacios R, de Amescua Seco A, O'Connor RV. The route to software process improvement in small-and medium-sized enterprises. In: Managing Software Process Evolution. Springer; 2016:109-136.

7. Larrucea X, O'Connor RV, Colomo-Palacios R, Laporte CY. Software process improvement in very small organizations. IEEE Softw. 2016;33(2):85-89.

8. Muñoz M., Mejia J., and Miramontes J., "Method for lightening software processes through optimizing the selection of software engineering best practices," in International Conference on Software Process Improvement, 2016, pp. 111-121.

9. O'Neill D. The way forward: a strategy for harmonizing agile and CMMI. CrossTalk. 2016;29(4):4-9.

10. Ambler SW, Lines M. The disciplined agile framework: a pragmatic approach to agile maturity. CrossTalk J Def Softw Eng. 2016;29(4):25-31.

11. Anwar A., Kamel A. A., and Ahmed E., "Agile adoption case study, pains, challenges \& benefits," in Proceedings of the 2nd Africa and Middle East Conference on Software Engineering, 2016, pp. 60-65.

12. Lehtinen TOA, Mäntylä MV, Vanhanen J, Itkonen J, Lassenius C. Perceived causes of software project failures - An analysis of their relationships. Inf Softw Technol. 2014;56(6):623-643.

13. Baddoo N, Hall T. Motivators of software process improvement: an analysis of practitioners' views. J Syst Softw. May 2002;62(2):85-96.

14. Yilmaz M, Yilmaz M, O'Connor RV, Clarke P. A gamification approach to improve the software development process by exploring the personality of software practitioners. In: Communications in Computer and Information Science. Vol.609; 2016:71-83.

15. Wilson DN, Hall T, Baddoo N. A framework for evaluation and prediction of software process improvement success. J Syst Softw. Nov. 2001;59(2): 135-142.

16. Passos OM, Dias-Neto AC, da Silva Barreto R. Organizational culture and success in SPI initiatives. leee Softw. 2012;29(3):97-99.

17. Niazi M. A comparative study of software process improvement implementation success factors. J Softw Evol Process. Sep. 2015;27(9):700-722.

18. Hamari J., Koivisto J., and Sarsa H., "Does gamification work?-a literature review of empirical studies on gamification," in 201447 th Hawaii International Conference on System Sciences, 2014, pp. 3025-3034.

19. Deterding S., Dixon D., Khaled R., and Nacke L., "From game design elements to gamefulness," in Proceedings of the 15th International Academic MindTrek Conference on Envisioning Future Media Environments-MindTrek'11, 2011, p. 9.

20. Zichermann G, Cunningham C. Gamification by Design: Implementing Game Mechanics in Web and Mobile Apps. O'Reilly Media; 2011.

21. Nah FF-H, Zeng Q, Telaprolu VR, Ayyappa AP, Eschenbrenner B. Gamification of Education: A Review of Literature. Cham: Springer; 2014:401-409.

22. Humphrey WS. Managing the Software Process. 1 MA: Addison-Wesley Reading; 1989.

23. Pino FJ, García F, Piattini M. Software process improvement in small and medium software enterprises: a systematic review. Softw Qual J. Jun. 2008; 16(2):237-261.

24. Staples M, Niazi M. Systematic review of organizational motivations for adopting CMM-based SPI. Inf Softw Technol. Jun. 2008;50(7-8):605-620.

25. Müller SD, Mathiassen L, Balshøj HH. Software Process Improvement as organizational change: a metaphorical analysis of the literature. J Syst Softw. Nov. 2010;83(11):2128-2146.

26. Ngwenyama $\mathrm{O}$, Nørbjerg $\mathrm{J}$. Software process improvement with weak management support: an analysis of the dynamics of intra-organizational alliances in IS change initiatives. Eur J Inf Syst. Jun. 2010;19(3):303-319.

27. Monteiro LFS, de Oliveira KM. Defining a catalog of indicators to support process performance analysis. J Softw Maint Evol Res Pract. Oct. 2011;23(6): 395-422.

28. Dyba T. An instrument for measuring the key factors of success in software process improvement. Empir Softw Eng. 2000;5(4):357-390.

29. Bayona-Oré S, Calvo-Manzano JA, Cuevas G, San-Feliu T. Critical success factors taxonomy for software process deployment. Softw Qual J. Mar. 2014;22(1):21-48.

30. Beecham S, Hall T, Rainer A. Software process improvement problems in twelve software companies: an empirical analysis. Empir Softw Eng. 2003; $8(1): 7-42$.

31. Iversen J. H., Mathiassen L., and Nielsen P. A., "Managing risk in software process improvement: an action research approach 1," 2004. 
32. Mathiassen L, Ngwenyama OK, Aaen I. Managing change in software process improvement. IEEE Softw. Nov. 2005;22(6):84-91.

33. Zahra K., Azam F., Ilyas F., Faisal H., Ambreen N., and Gondal N., "Success factors of organizational change in software process improvement," in Proceedings of the 5th International Conference on Information and Education Technology-ICIET'17, 2017, pp. 155-160.

34. O'Connor R, Basri S. The effect of team dynamics on software development process improvement. Int $J$ Hum Cap Inf Technol Prof. 2012;3(3):13-26.

35. Abrahamsson P, livari N. Commitment in software process improvement-in search of the process. Proc Annu Hawaii Int Conf Syst Sci. 2002; 2002(January):3239-3248.

36. Pereira P., Duarte E., Rebelo F., and Noriega P., "A review of gamification for health-related contexts," in International conference of design, user experience, and usability, 2014, pp. 742-753.

37. Witt M., Scheiner C. W., and Robra-Bissantz S., "Gamification of online idea competitions: insights from an explorative case., in GI-Jahrestagung, 2011, p. 392.

38. Khandelwal S., Sripada S. K., and Reddy Y. R., "Impact of gamification on code review process: an experimental study," in Proceedings of the 10th Innovations in Software Engineering Conference, 2017, pp. 122-126.

39. Pedreira O, García F, Brisaboa N, Piattini M. Gamification in software engineering-a systematic mapping. Inf Softw Technol. 2015;57:157-168.

40. Petersen K, Vakkalanka S, Kuzniarz L. Guidelines for conducting systematic mapping studies in software engineering: an update. In: Information and Software Technology. Vol.64; 2015:1-18.

41. Wieringa R, Maiden N, Mead N, Rolland C. Requirements engineering paper classification and evaluation criteria: a proposal and a discussion. Requir Eng. 2006;11(1):102-107.

42. Werbach K, Hunter D. For the Win: How Game Thinking Can Revolutionize Your Business. Wharton; 2012.

43. Cruzes DS, Dyba T. Recommended steps for thematic synthesis in software engineering. Int Symp Empir Softw Eng Meas. $2011 ;(7491): 275-284$.

44. Chou Y. K., "Octalysis: complete gamification framework-Yu-kai Chou." [Online]. Available: https://yukaichou.com/gamification-examples/octalysiscomplete-gamification-framework/. [Accessed: 12-Dec-2018].

45. Alhammad MM, Moreno AM. Gamification in software engineering education: a systematic mapping. J Syst Softw. Jul. 2018;141:131-150.

46. Alhammad M. M. and Ana M. Moreno, "What is going on in agile gamification (extended abstract)," in Proceedings of the 19th International Conference on Agile Software Development: Companion. ACM, 2018.

47. Buisman A. L. D. and Van Eekelen M. C. J. D., "Gamification in educational software development," in Proceedings - CSERC 2014: Computer Science Education Research Conference, 2014, pp. 9-20.

48. Robertson M., "Can't play, won't play," 2010. [Online]. Available: https://kotaku.com/5686393/cant-play-wont-play. [Accessed: 11-Dec-2018].

\section{APPENDIX: LIST OF PRIMARY STUDIES A.}

[P1]. Dorling, A. and McCaffery, F. (2012) 'The gamification of SPICE', in International Conference on Software Process Improvement and Capability Determination. Springer, pp. 295-301.

[P2]. Herranz, E. and Colomo-Palacios, R. (2018) 'Is gamification a way to a softer software process improvement? A preliminary study of success factors', in European Conference on Software Process Improvement. Springer, pp. 207-218.

[P3]. Herranz, E. et al. (2014) 'Gamification as a disruptive factor in software process improvement initiatives', J. UCS, 20(6), pp. 885-906.

[P4]. Herranz, E. et al. (2016) 'Towards a gamification framework for software process improvement initiatives: construction and validation', Journal of Universal Computer Science, 22(12), pp. 1509-1532. Available at: http://www.jucs.org/jucs_22_12/towards_a_gamification_ framework/jucs_22_12_1509_1532_herranz.pdf.

[P5]. Herranz, E., Colomo-Palacios, R. and Al-Barakati, A. (2017) 'Deploying a gamification framework for software process improvement: preliminary results', in European Conference on Software Process Improvement. Springer, pp. 231-240.

[P6]. Herranz, E., Colomo-Palacios, R. and Seco, A. de A. (2015) 'Gamiware: a gamification platform for software process improvement', in Communications in Computer and Information Science. doi: 10.1007/978-3-319-24647-5_11.

[P7]. Ruiz, M., Trinidad, M. and Calderón, A. (2016) 'Gamification and functional prototyping to support motivation towards software process improvement', in International Conference on Product-Focused Software Process Improvement. Springer, pp. 697-704.

[P8]. Yilmaz, M. and O'Connor, R. V (2016) 'A scrumban integrated gamification approach to guide software process improvement: a turkish case study', Tehnicki Vjesnik, 23(1), pp. 237-245. 